\title{
Approach to Managing Febrile Patients in the ICU
}

\author{
Dibyendu Banerjee $^{1^{*}}$, Tanushree Banerjee ${ }^{2}$ and Vivek Vidyadhar Bhosale ${ }^{1^{*}}$ \\ ${ }^{1}$ CSIR-Central Drug Research Institute, Janakipuram Extension, Sitapur Road, Lucknow, India \\ ${ }^{2}$ IAMR (Indian Academy of Medical Research), Gaziabad, India
}

"Corresponding authors: Dibyendu Banerjee, CSIR-Central Drug Research Institute, Janakipuram Extension, Sitapur Road, Lucknow, India, Tel: 91-1105-222772450; Fax: 911105222771941; E-mail: d.banerjee@cdri.res.in

Vivek Vidyadhar Bhosale, CSIR-Central Drug Research Institute, Janakipuram Extension, Sitapur Road, Lucknow, India, Tel: 91-0522-2772450; Fax: 91-0522-2772450;

E-mail: drvivekbhosale@cdri.res.in

Rec date: July 1, 2016; Acc date: October 19, 2016; Pub date: October 26, 2016

Copyright: (c) 2016 Banerjee D, et al. This is an open-access article distributed under the terms of the Creative Commons Attribution License, which permits unrestricted use, distribution, and reproduction in any medium, provided the original author and source are credited.

\begin{abstract}
Background and Aims: A febrile response is a common phenomenon in the ICU (Intensive Care Unit), often with complex and unknown origins. Fever is only one component of a febrile response and may or may not be an indication of severe infection.

Subjects and Methods: A febrile response caused by severe infection/s is often accompanied by neutropenia where the absolute neutrophil count goes below $500 / \mathrm{mm}^{3}$, or is predicted to go below $500 / \mathrm{mm}^{3}$ over the next 48 hours. Febrile neutropenia is generally an indication for an infection requiring the commencing of empirical antibiotics after taking appropriate cultures. However, severe infections may also occur in the absence of fever or neutropenia.

Conclusion: In cases of new fever, or fever of unknown origin, it is highly recommended that the latest IDSA (Infectious Disease Society of America) or SCCM (Society of Critical Care Medicine) guidelines be followed. Some recently launched antimicrobials not yet covered by the IDSA are discussed and maybe useful for treatment of drug resistant cases. It may also be useful to remember that fever is more often a friend than a foe.
\end{abstract}

\section{Introduction}

Fever is a normal adaptive response of the body to infectious and non-infectious causes involving a cytokine-mediated response, the generation of acute phase reactants, and the activation of numerous physiologic, endocrinologic and immunologic systems. In the intensive care unit (ICU), fever is a very common occurrence. percent of medical patients are known to become febrile during their hospitalization, while up to $90 \%$ of critically ill patients with severe sepsis are known to experience fever during their stay in the ICU $[1,2]$. About half of all newly detected febrile episodes are of non-infectious origin. In the ICU, fever should be treated in cardiorespiratory and neurosurgical patients and in those in whom temperature exceeds $40^{\circ} \mathrm{C}$ $\left(104^{\circ} \mathrm{F}\right)$ [3]. Antipyretic therapy must be regardless of the metabolic cost, the result and the side \$presence of fever

Wheads to an array of diagnostic tests and procedures with increased medical costs and exposure of patients to antibiotics. Most importantly, the inappropriate use of antibiotics at this stage can result in antibiotic resistance. ICU requires a meticulous, attentive and a more restrictive approach than simply starting antibiotic therapy. In this review, we dicuss the complex causes and pathophysiology of a febrile response and then go on to discuss the best approaches for management of the febrile response for patients in the ICU.

\section{Pathophysiology of a febrile response}

đfebrile response is a complex pathophysiological response to disease. It includes the activation of various physiologic, immunologic and endocrinologic systems [4-6]. Fever, which usually is only one component of the febrile response, is a single oral temperature measurement of $101^{\circ} \mathrm{F}$ or higher, or a temperature of $100.4^{\circ} \mathrm{F}$ or higher sustained over a 1 hour period [7]. Recommendations of the Society of Critical Care Medicine (SCCM) and Infectious Disease Society of America (IDSA) formulate that any new fever in the ICU should be investigated only if the temperature is $101^{\circ} \mathrm{F}$ or greater [7].

\section{Causes, diagnostic approach and treatment of fever in the ICU}

Critically ill patients frequently show a single spike of elevated temperature that generally returns to normal without any treatment being खvents have no clinical and are related to in erventions, such as blood transfusion, placement of a uninary catheter or endotracheal suctioning, etc. Any fever that is related to an invasive procedure and without transient bacteremia, frequently self dissipates, whereas fever caused by underlying chronic diseases, or medical complications, or reactions to drug therapy may require intervention. đollowing sections cover the febrile response in both infectious and non-infectious scenarios. A list of the commonly observed causes of fever in the ICU are listed in Table 1. 


\begin{tabular}{|c|c|c|}
\hline Infected body part & Cause of infection & Non-infectious Causes \\
\hline $\begin{array}{l}\text { Infections of the Central } \\
\text { Nervous System (CNS) }\end{array}$ & Meningitis, encephalitis & $\begin{array}{l}\text { Posterior fossa syndrome, central fever, seizures, cerebral infraction, hemorrhage, } \\
\text { cerebrovascular accident }\end{array}$ \\
\hline $\begin{array}{l}\text { Infections of the } \\
\text { Cardiovascular System }\end{array}$ & $\begin{array}{l}\text { Central line, infected pacemaker, endocarditis, } \\
\text { sterna osteomyelitis, viral-pericarditis }\end{array}$ & $\begin{array}{l}\text { Myocardial infarction, myocardial/ perivalvular abscess, balloon pump syndrome, post- } \\
\text { pericardiectomy syndrome }\end{array}$ \\
\hline $\begin{array}{l}\text { Infections of the } \\
\text { Pulmonary System }\end{array}$ & $\begin{array}{l}\text { VAP, mediastinitis, tracheobronchitis, } \\
\text { empyema }\end{array}$ & $\begin{array}{l}\text { Pulmonary emboli, ARDS, atelectasis (without pneumonia), BOOP, bronchogenic } \\
\text { carcinoma without postobstructive pneumonia, systemic lupus erythemaosus } \\
\text { pneumonitis }\end{array}$ \\
\hline $\begin{array}{l}\text { Infections of the Gastro- } \\
\text { Intestinal (GI) tract }\end{array}$ & $\begin{array}{l}\text { Intra-abdominal abscess, cholangiitis, } \\
\text { cholecystitis, viral hepatitis, peritonitis, diarrhea } \\
\text { (Clostridium difficile) }\end{array}$ & $\begin{array}{l}\text { Pancreatitis, acalculus cholecystitis, ischemia of the bowel, bleeding, cirrhosis, ischemic } \\
\text { colitis, irritable bowel syndrome }\end{array}$ \\
\hline $\begin{array}{l}\text { Infections of the Urinary } \\
\text { tract }\end{array}$ & $\begin{array}{l}\text { Bacteremia, urosepsis, pyelonephritis,cystitis } \\
\text { associated with the catheter }\end{array}$ & \\
\hline $\begin{array}{l}\text { Infections of the Skin/soft } \\
\text { tissue }\end{array}$ & Wound infections, decubitus ulcers, cellulitis & -- \\
\hline $\begin{array}{l}\text { Infections of the Bone/ } \\
\text { joint }\end{array}$ & Arthritis, chronic osteomyelitis & Acute gout \\
\hline Other causes & Transient bacteremia, sinusitis, etc. & $\begin{array}{l}\text { Postoperative fever }(48 \mathrm{~h}) \text {, fever after transfusion, drug fever, adrenal insufficiency, } \\
\text { phlebitis/ thrombo-phlebitis, neoplastic fever, alcohol/drug withdrawal, delirium tremens, } \\
\text { deep venous thrombosis }\end{array}$ \\
\hline
\end{tabular}

Table 1: Commonly observed infective and non-infective agents causing fever in the ICU [3].

\section{Febrile response in the ICU caused by infectious agents}

Infections in the ICU occur at a rate of between $10 \%$ and $20 \%$ (according to NNIS and EPIC studies respectively). Ventilatorassociated pneumonia (VAP) is the most common, followed by sinusitis, bloodstream and catheter-related infections. Dare followed in frequency by nosocomial diarrhoea and wound infections $[8,9]$. Primary evaluation includes: (i) a chest radiograph and CT scan; (ii) cultures of secretions from the lower respiratory tract before antibiotics administration (expectorated sputum, tracheal secretions, broncho-alveolar lavage BAL obtained by bronchoscopy); and (iii) in case of pleural stin culture and cytology of the pleural \$10,11]. Blood cultures and other blood tests (PCR, CMV antigen, galactomannan and beta-D-glucan) can also add value to the diagnostic procedure $[7,11]$.

\section{Fever due to intravascular devices}

冈majority of ICU patients have one or more central venous catheters (CVC) inserted into their bodies. Many of them might also have a tunnelled or $\quad$ CVC, or subcutaneous central venous port. Hence catheter related infections caused by pathogens that colonize a vascular device are always a possibility in the ICU [3]. According to recorded literature, such infections are seen at an incidence of about 10 infections/ 1000 catheter days, while the relative risk for their appearance depends on various parameters such as the period the catheter is present in situ, the number of ports it has, the number of manipulations that have been done, the kind of device, and the techniques used in insertion [10]. \diagnosis is based on clinical signs including: the of drawing or infusing through the catheter, the presence of at the insertion site, and the recovery of microorganisms in multiple blood cultures [11]. For the evaluation of those signs, two peripheral blood cultures or one drawn percutaneously and one drawn through the catheter should be obtained [12]. 伍ccepted standard for the diagnosis and treatment of such infections is the culture of the catheter itself with semiquantitative or quantitative catheter tip methods [13].

\section{Sinusitis}

Nosocomial sinusitis is an infection more common to closed and spaces. are not very frequent but can have serious consequences upon occurrence [14,15]. \predisposing factors for the development of sinusitis include: nasotracheal or nasogastric tube placement, nasal packing, facial fractures, and steroid administration.

Wiagnosis is made by radiographs, CT scans or magnetic resonance imaging (MRI) of the sinus [14]. Nasal endoscopy, in conjunction with plain radiography may also increase the accuracy of diagnosis by a skilled practitioner $[16,17]$. Sinusitis occurs at the rate of about $5 \%$ of all nosocomial infections in the ICU minly neurosurgical patients, characterized by fever and leykocytosis, while purulent nasal discharge is acking (present in no more than $25 \%$ of proven cases).

\section{Fever due to nosocomial diarrhoea}

Many patients in the ICU manifest diarrhoea as more than two stools per day that takes the shape of the container when placed in it). Diarrhoea can be caused by enteral feeding or by drugs [18]. $\quad$ most common cause of febrile diarrhoea in critically ill patients is Clostridium (\$mprising 10\%-25\% of all cases of antibioticassociated diarrhoea). All patients with fever or leukocytosis and diarrhoea, who receive an antibacterial agent or chemotherapy within 60 days before the onset of diarrhoea, should be suspected of carrying a $C$ inflection [18-20]. \standard diagnosis involves the tissue culture assay, which presents, however, a 24-48 hour delay in results and high cost, while an enzyme immunoassay test (EIA) for toxin $\mathrm{A}$ and $\mathrm{B}$ is commercially available, easy to perform, and able to 
provide results within few minutes to a couple of hours [21]. In case of severe illness and negative rapid tests for $C$.,$\nabla$ sigmoidoscopy procedure remains a secure option for the diagnosis.

\section{Fever due to intra-abdominal and surgical site infections}

Intra-abdominal or surgical site infections are the main causes of ICU admission due to abscess formation or biliary sepsis). $\bigotimes$ diagnosis is facilitated by CT scan of the abdomen, ultrasound and nuclear medicine techniques (gallium-67, indium-111 white blood cell scintigraphy) [3,15]. Surgical site infections include mainly the contamination of the surgical incision depending on the medical co morbidities of the patient, the duration of the operation, and whether antimicrobial prophylaxis was administered before incision [22]. It may be required to open, debride and pack the wound along with other measures such as percutaneous or operative drainage and the use of antibiotics [23].

\section{Fever due to urinary tract infections}

Urinary tract infections (UTIs) in critically ill patients (mainly catheter-associated bacteriuria or candiduria) usually colonization, are rarely symptomatic, and are not considered as a ca将e of morbidity or attributable mortality. đraditional clinical symptoms include dysuria, urgency, pelvic or fever or chills. However, these symptoms are rarely reported in patients in the ICU with documented catheter-associated bacteriuria or candiduria [24-28]. In the ICU, Gram-negative bacilli other than $E$. coli, Enterococcus species, and yeasts are the major causes of urinary tract infections $[25,28]$. To unambiguously prove that the urinary tract is the source of fever, a specimen of urine should be obtained directly from the catheter sampling port and evaluated by microscopy, gramnegative stain, and quantitative culture.

\section{Fever due to fungal infections}

Fungi (mainly Candida species) are a major cause of infections and constitute a very large percentage of nosocomial fungal infections in critically ill patients. It is associated with risk factors and is characterized by diagnosis due to lack of diagnostic tools and the ability to discriminate between colonization and infection. In the last few years, fungal infections have shown a distinct tखwards non-albicans species. $\quad \nabla \quad$ diagnosis is made by the of the fungi from histologic or, sterile specimens obtained from the patient [29-32].

\section{Fever due to viral infections}

In recent years, infection and antigenic response to cytomegalovirus (CMV) has been proposed as a cause of unexplained and prolonged fever in severely ill, immune-competent patients in the ICU, but the of CMV detection is unknown. A high morbidity and mortality rate is seen in patients with a CMV infection compared with patients in whom the virus is not undetected [33]. Herpes simplex virus (HSV) or varicella-zoster virus (VZV) infection are also possible but must be treated for only upon proper diagnosis.

\section{Fever due to infections of the CNS}

In neurocritical patients, fever occurs in $25 \%$ of the cases and almost half of these fevers are non-infectious in nature. In these patients, the suspicion of infection development must be of high index because of the inherent limitations of the neurologic examination, the low yield of lumbar puncture in non immune-compromised patients, and the contraindications for lumbar puncture performance, which frequently are met in critically ill patients $[33,34]$. In these patients, the diagnosis for a critically ill patient with a new episode of fever is usually done by imaging study (CT scan of the brain), culture of cerebrospinal and removal and culture of the placed catheter or other intracranial device $[35,36]$.

\section{Febrile response in the ICU caused by non-infectious agents}

Half of the episodes of fever in the ICU belong to the non-infectious category, with temperatures not usually exceeding $38.3^{\circ} \mathrm{C}$ [37]. Experience suggests that clinicians are aided in a diagnosis by carefully studying the medical history, carefully charting the recent interventions and by conducting a thorough physical examination of the patient. However, the type of ICU population (e.g., medical, surgical, trauma, neurosurgery and burn patients), the type of patients (e.g., immune-compromised, elderly, etc.), the history of recent epidemics and the local epidemiology, must also be taken into $\nabla$ account [18]

\section{Post-operative fever in the ICU}

Fever is a common phenomenon during the hrs $\mathbb{Q}$ surgery. However, the silver lining is that this kind of early postoperative fever is usually noninfectious in origin [38]. However, if the fever persists even 96 hours post-operation, the most likely cause for such fever is an infection. Recommendations for evaluation of fever within 72 hours of surgery:

\$urgical site must be examined daily for infections. A culture is not required or suggested unless there are any obvious signs of infection.

In patients who are sedentary, have lower limb immobility, have a malignant neoplasm, or are taking an oral contraceptive, a high level of suspicion should be maintained for deep venous thrombosis, thrombophlebitis, and pulmonary embolism.

A urine analysis or culture or a chest radiograph is not necessary during the 7 post-operation if fever is the only indication.

\section{Drug induced fever in the ICU}

About 3\%-7\% of febrile episodes are attributable to reactions caused by drugs. Fever caused by drug hypersensitivity or drug-related fever or "drug fever" are to खdiagnose and usually established by exclusion rather than a diagnosis [25]. Several drugs can cause fever due to hypersensitivity and local at the site of administration or due to intra-venous and intra-vascular delivery systems [18]. Tell-tale signs such as the appearance of a maculopapular rash, an increased WBC count and a moderate elevation of serum transaminases are rarely accompanied with such fever (less than 5-10\% of cases) whereas peripheral eosinophilia and a markedly elevated erythrocyte sedimentation rate $(>100 \mathrm{~mm} / \mathrm{h})$ maybe recorded [18]. $\otimes \quad$ accompanying signs associated with drug-fever are a lack of appropriate pulse rate response and a relative bradycardia. $\quad \nabla$ high-risk agents for drug-fever include all antibiotics (especially blactams), anti-epileptic drugs (especially phenytoin), antiarrhythmics (mainly quinidine and procainamide), antihypertensives (amethyldopa), diuretics, anti-seizure drugs, and stool [\$39]. Low risk antibiotics include clindamycin, vancomycin, 
chloramphenicol, aztreonam, doxycycline, erythromycin, imipemen, quinolones, and aminoglycosides [3,39,40]. $\nabla$ estimated time between initiating a drug and the appearance of fever is 21 days (median 8 days) while the fever usually subsides within 72 hours of drug removal. When present with the fever, a rash may persist for days or weeks $[3,18,39]$.

\section{Guideline IDSA Recommendations for Evaluation and Treatment of Patients with Fever and Neutropenia}

$\otimes$ remmendation tries to distinguish the high and low-risk patients with fever and neutropenia. According to the guidelines [7]:

Patients with anticipated prolonged ( $>7$ days duration) and profound neutropenia (neutrophil count ANC $<100$ cells $/ \mathrm{mm}^{3}$ ) are categorized as high risk. are usually accompanied by medical co-morbid conditions, including hypotension, pneumonia, new-onset abdominal pain, or neurologic changes. High-risk patients have a MASCC score $<21$.

Patients with anticipated brief ( $<7$ days duration) neutropenic periods or no or few comorbidities, are considered to be low-risk. Carefully selected low-risk patients may be candidates for oral and/or outpatient empirical antibiotic therapy. Low-risk patients have a MASCC score $>21$.

Tests and Cultures to be performed during the initial assessment. Recommendations include [7]:

A complete blood cell (CBC) count with

leukocyte count and platelet count. Measurements of serum levels of creatinine, blood urea nitrogen, electrolytes, hepatic transaminase enzymes and total bilirubin are also recommended.

A minimum of two sets of blood cultures, collected simultaneously from each lumen of a central venous catheter (CVC) if present, or from a peripheral vein site, are recommended. Two blood cultures from separate venipunctures must be taken if no central catheter is present.

If other infection sites are clinically indicated or suspected, then culture specimens from these sites must also be collected.

In patients with indicated respiratory signs or symptoms, a chest radiograph is highly recommended.

Apart from IDSA guidelines, testing some people for Procalcitinin (specially those fr囚m respiratory infections) may be useful to detect bacterial infections. 凶serum level of procalcitonin goes up with bacterial replication and goes down when they stop replicating, hence providing indications as to when to start or stop antibiotics. A

study by Schuetz and colleagues included more than 4000 patients in randomized trials [41]. Ðresearchers examined the use of procalcitonin and found a very useful take-home message: If you have a patient with a pulmonary and you have procalcitonin, you are going to use antibiotics, probably regardless of the actual procalcitonin level. Nesearchers showed that physicians are going to use the antibiotic anyway, but it will help them know when to stop.

Empiric antibiotic therapy appropriate for febrile patients with neutropenia. Recommendations include [7]:

High-risk hospitalized patients receive intravenous empirical antibiotic therapy. In case antimicrobial resistance is suspected or proven, therapy with an antipseudomonal b-lactam agent such as cefepime, acarbapenem (meropenem or imipenem-cilastatin), or piperacillin-tazobactam, and other antimicrobials like aminoglycosides, to the initial regimen. an $\mathbb{W} /$ or vancomycin may be added well as helps manage complications like hypotension and pneumonia.

In cases of clinical indications such as suspected catheterrelated infection, skin or inflection, pneumonia, or hemodynamic instability, antibiotics like vancomycin or others that are active against aerobic gram-positive cocci are recommended. are however not part of the standard initial antibiotic regimen for fever and neutropenia.

囚empirical therapy may be for infections with antibiotic-resistant organisms. For example:

i. For MRSA (Methicillin-resistant Staphylococcus aureus): early addition of vancomycin, linezolid, or daptomycin is advised.

$\nabla$ ii. For VRE (Vancomycin-resistant Enterococcus): early addition of linezolid or daptomycin is advised.

iii. For ESBLs (Extended Spectrum Beta Lactamase): early use of carbapenem is advised.

iv. For KPCs (Klebsiella pneumoniae carbapenemase): early use of polymyxin-colistin or tigecycline is advised.

For low-risk patients who are not anticipated to remain neutropenic for $>7$ days, antibacterial prophylaxis is not recommended.

\section{Treatment of superbugs with newer antimicrobials}

囚US Food and Drug Administration (FDA) advisory board has recently approved two new drugs which will be very useful for treatment of patients fr囚m drug resistant infections. Tedizolid phosphate (Sivextro"') is a drug that is very much like linezolid except that it is given once a day. Dalbavancin (Dalvance") is an interesting drug that has a half-life of 6.5 days. Once injected the patient is covered for a week, and tøe second dose the patient gets 2 weeks of therapy. has some unique properties in terms of simplicity of use and compliance. Last year, US-FDA approved another drug called Telavancin (Vibativ"') which has proven to be very agdinst hospital-acquired and ventilator-associated pneumonia [42].

\section{The conserved nature of the febrile response}

đebrile response has been conserved for millions of years among members of the animal kingdom, both vertebrates and invertebrates. Upon challenge with bacteria, fever Melps the host get rid of the invading pathogens [43]. fever has been used in the preantibiotic era, with limited success to treat neurosyphilis [44]. However, controlled studies evaluating the of fever in humans do not exist. Data from in vitro studies and animal studies suggest that fever may be to the host $[1,45]$. In fact, in one recent review, two-thirds of the studies (14 out of 21) demonstrated a deleterious of lowering body temperature [46]. Other studies have highlighted the o $\$$ temperature on the minimum inhibitory concentration of antibiotics to bacteria. At temperatures greater than $38.5^{\circ} \mathrm{C}$, the authors of one study reported a progressive increase in the antimicrobial activity of antibiotics [47] It is therefore widely believed that a physiology so widely preserved must convey some net t\$ the host; otherwise it would not have been retained in evolution despite its high metabolic cost to the host $[44,48]$. 
Page 5 of 6

\section{Concluding Remarks}

In conclusion, a structured approach is necessary to properly diagnose and treat fever in the ICU. 叉nature and duration of antibiotic therapy is dictated by the kind of infection and the site of infection. If neutropenia is seen, the appropriate antibiotics must continue atleast until neutropenia lasts (i.e., until Absolute Neutrophil Count (ANC) is $>500$ cells $/ \mathrm{mm}^{3}$ ) or longer if clinically necessary. In cases of unexpected or unexplained fever, the initial antibiotic regimen must be continued until there are clear signs of marrow recovery; the traditional endpoint is an increasing ANC that exceeds $500 \mathrm{cells} / \mathrm{mm}^{3}$ [49]. However, when an appropriate treatment course has been completed and all signs and symptoms of a documented infection have resolved, patients who are still neutropenic may require an oral p phylaxis until full recovery. \high fever in ICU was associated with inc殴ased risk for death 20.3\% [50]. Use of practising guidelines and rational therapy may reduce the mortality and morbidity. Finally, it is important to remember that fever has myriad immune-stimulatory the maybe the patient and it is sometimes prudent to remember the old aphorism when treating it: Primum non nocere do no harm).

\section{Acknowledgements}

דuthors would like to acknowledge CSIR-CDRI for the support and logistics needed in writing this manuscript. Qtemporary manuscript number provided by CSIR-CDRI is 246/2014/DB.

\section{References}

1. Cunha BA (1998) Fever in the critical care unit. Crit Care Clin 14: 1-14.

2. Cunha BA, Shea KW (1999) Fever in the intensive care unit. Infect Dis Clin North Am 10: 185-209.

3. Dimopoulos G, Falagas ME (2009) Approach to the febrile patient in the ICU. Infect Dis Clin North Am 23: 471-484.

4. Dalal S, Zhukovsky DS (2006) Pathophysiology and management of fever. J Support Oncol 4: 9-16.

5. Mackowiak PA (2006) Pathophysiology and management of fever-we know less than we should. J Support Oncol 4: 21-22.

6. Robins HI, Brandt K, Longo WL (2006) Pathophysiology and management of fever revisited. J Support Oncol 4: 265-266.

7. Freifeld AG, Bow EJ, Sepkowitz KA, Boeckh MJ, Ito JI, et al. (2011) Clinical practice guideline for the use of antimicrobial agents in neutropenic patients with cancer: 2010 update by the infectious diseases society of america. Clin Infect Dis 52: e56-93.

8. Jarvis WR, Edwards JR, Culver DH, Hughes JM, Horan T, et al. (1991) Nosocomial infection rates in adult and pediatric intensive care units in the United States. National Nosocomial Infections Surveillance System. Am J Med 91: 185S-191S.

9. Vincent JL, Bihari DJ, Suter PM, Bruining HA, White J, et al. (1995) \ prevalence of nosocomial infection in intensive care units in Europe. Results of the European Prevalence of Infection in Intensive Care (EPIC) Study. EPIC International Advisory Committee. JAMA 274: 639-644.

10. Canadian Critical Care Trials Group (2006) A randomized trial of diagnostic techniques for ventilator-associated pneumonia. N Engl J Med 355: 2619-2630.

11. Rello J, Diaz E, Rodriguez A (2005) Advances in the management of pneumonia in the intensive care unit: review of current thinking. Clin Microbiol Infect 11: 30-38.

12. Safdar N, Maki DG (2002)

at the insertion site is not predictive of catheter-related bloodstream infection with short-term 遍ntral venous catheters. Crit Care Med 30: 2632-2635.
13. Catton JA, Dobbins BM, Kite P, Wood JM, Eastwood K, et al. (2005) In situ diagnosis of intravascular catheter-related bloodstream infection: a comparison of quantitative culture time to positivity and endoluminal brushing. Crit Care Med 33: 787-791.

14. Vargas F, Bui HN, Boyer A, Bébear CM, Lacher-Fougére S, et al. (2006) Transnasal puncture based on echographic sinusitis evidence in mechanically ventilated patients with suspicion of nosocomial maxillary sinusitis. Intensive Care Med 32: 858-866.

15. Marik PE (2000) Fever in the ICU. Chest 117: 855-869.

16. Maclennan AC, Jones NS (1995) 区diagnosis of disease. J Laryngol Otol 109: 1130-1131.

17. Roberts DN, Hampal S, East CA, Lloyd GA (1995) 区diagnosis of sinonasal disease. J Laryngol Otol 109: 27-30.

18. O'Grady NP, Barie PS, Bartlett JG, Bleck T, Carroll K, et al. (2008) Guidelines for evaluation of new fever in critically ill adult patients: 2008 update from the American College of Critical Care Medicine and the Infectious Diseases Society of America. Crit Care Med 36: 1330-1349.

19. Bartlett JG (2006) Narrative review: the new epidemic of Clostridium enteric disease. Ann Intern Med 145: 758-764.

20. Pépin J, Valiquette L, Alary ME, Villemure P, Pelletier A, et al. (2004) Clostridium diarrhea in a region of Quebec from 1991 to 2003: a changing pattern of disease severity. CMAJ 171: 466-472.

21. Ticehurst JR, Aird DZ, Dam LM, Borek AP, Hargrove JT, et al. (2006) detection of toxigenic Clostridium by凶a two-step algorithm including tests for antigen and cytotoxin. J Clin Microbiol 44: 1145-1149.

22. Stevens DL, Bisno AL, Chambers HF, Everett ED, Dellinger P, et al. (2005) Practice guidelines for the diagnosis and management of skin and $\square$ tissue infections. Clin Infect Dis 41: 1373-1406.

23. Kothari VM, Karnad DR (2005) New onset fever in the intensive care unit. J Assoc Physicians India 53: 949-953.

24. Safdar N, Slattery WR, Knasinski V, Gangnon RE, Li Z, et al. (2005) Predictors and outcomes of candiduria in renal transplant recipients. Clin Infect Dis 40: 1413-1421.

25. Tambyah PA, Maki DG (2000) Catheter-associated urinary tract infection is rarely symptomatic: a prospective study of 1497 catheterized patients. Arch Intern Med 160: 678-682.

26. Gaynes R, Edwards JR (2005) Overview of nosocomial infections caused by gram-negative bacilli. Clin Infect Dis 41: 848-854.

27. Klevens RM, Edwards JR, Tenover FC, McDonald LC, Horan T, et al. (2006) Changes in the epidemiology of methicillin-resistant Staphylococcus aureus in intensive care units in US hospitals, 1992-2003. Clin Infect Dis 42: 389-391.

28. Laupland KB, Bagshaw SM, Gregson DB, Kirkpatrick AW, Ross T, et al. (2005) Intensive care unit-acquired urinary tract infections in a regional critical care system. Crit Care 9: R60-65.

29. Dimopoulos G, Karabinis A, Samonis G, Falagas ME (2007) Candidemia in immunocompromised and immunocompetent critically ill patients: a prospective comparative study. Eur J Clin Microbiol Infect Dis 26: 377-384.

30. Dimopoulos G, Ntziora F, Rachiotis G, Armaganidis A, Falagas ME (2008) Candida albicans versus non-albicans intensive care unit-acquired bloodstream infections: Analg 106: 523-529.

31. Dimopoulos G, Velegraki A, Pefanis A, Karabinis A (2007) Candiduria in critically ill patients: an early candidemia marker when coupled with multiple blood specimens PCR assays? Hepatogastroenterology 54: 354-358.

32. Holley A, Dulhunty J, Blot S, Lipman J, Lobo S, et al. (2009) Temporal trends risk factors and outcomes in albicans and non-albicans candidaemia: an international epidemiological study in four multidisciplinary intensive care units. Int J Antimicrob Agents 33: e1-7.

33. Jaber S, Chanques G, Borry J, Souche B, Verdier R, et al. (2005) Cytomegalovirus infection in critically ill patients: associated factors and consequences. Chest 127: 233-241. 
Citation: Banerjee D, Banerjee T, Bhosale VV (2016) Approach to Managing Febrile Patients in the ICU. Gen Med (Los Angeles) 4: 274. doi: 10.4172/2327-5146.1000274

Page 6 of 6

34. Commichau C, Scarmeas N, Mayer SA (2003) Risk factors for fever in the neurologic intensive care unit. Neurology 60: 837-841.

35. Sinner SW, Tunkel AR (2004) Antimicrobial agents in the treatment of bacterial meningitis. Infect Dis Clin North Am 18: 581-602.

36. Tunkel AR, Hartman BJ, Kaplan SL, Kaufman BA, Roos KL, et al. (2004) Practice guidelines for the management of bacterial meningitis. Clin Infect Dis 39: 1267-1284.

37. Cunha BA (1994) Intensive care not intensive antibiotics. Heart Lung 23: 361-362.

38. Garibaldi RA, Brodine S, Matsumiya S, Coleman M (1985) Evidence for the non-infectious etiology of early postoperative fever. Infect Control 6: 273-277.

39. Wood AJ (1998) Harrison's principles of internal medicine. McGrawhill. pp: $422-430$.

40. Kiekkas P, Brokalaki H, Manolis E, Askotiri P, Karga M (2007) Fever and standard monitoring parameters of ICU patients: a descriptive study. Intensive Crit Care Nurs 23: 281-288.

41. Schuetz P, Chiappa V, Briel M, Greenwald JL (2011) Procalcitonin algorithms for antibiotic therapy decisions: a systematic review of randomized controlled trials and recommendations for clinical algorithms. Arch Intern Med 171: 1322-1331.
42. John G, Bartlett ID (2014) New threats, old antibiotics.

43. Mackowiak PA (1994) Fever: blessing or curse? A unifying hypothesis. Ann Intern Med 120: 1037-1040.

44. Ryan M, Levy MM (2003) Clinical review: fever in intensive care unit patients. Crit Care 7: 221-225.

45. Bruderlein U, Strupp P, Vagts DA (2006) Fever in intensive care patients. Anasthesiol Intensivmed Notfallmed Schmerzther 41: E8-E18.

46. Kluger MJ, Kozak W, Conn CA, Leon LR, Soszynski D (1996) 区 adaptive value of fever. Infect Dis Clin North Am 10: 1-20.

47. Mackowiak PA, Marling-Cason M, Cohen RL (1982) هf temperature on antimicrobial susceptibility of bacteria. J Infect Dis 145: 550-553.

48. Zitelli BJ (1991) Fever phobia and the adaptive value of fever. Indian J Pediatr 58: 275-278.

49. Cunha BA (1996) Fever of unknown origin. Infect Dis Clin North Am 10: 111-117.

50. Laupland KB, Shahpori R, Kirkpatrick AW, Ross T, Gregson DB, et al. (2008) Occurrence and outcome of fever in critically ill adults. Crit Care Med 36: 1531-1535. 